\title{
Caring about bone-modifying agents (BMAs) in women with early-stage breast cancer
}

\author{
Charles L. Shapiro \\ Icahn School of Medicine at Mt Sinai, New York, NY, USA \\ Correspondence to: Charles L. Shapiro. Icahn School of Medicine at Mt Sinai, New York, NY 10029, USA. Email: charles.shapiro@mssm.edu. \\ Provenance and Peer Review: This article was commissioned by the editorial office, Annals of Translational Medicine. The article did not undergo \\ external peer review. \\ Comment on: Coleman R, Finkelstein DM, Barrios C, et al. Adjuvant denosumab in early breast cancer (D-CARE): an international, multicentre, \\ randomised, controlled, phase 3 trial. Lancet Oncol 2020;21:60-72.
}

Submitted Feb 29, 2020. Accepted for publication Mar 24, 2020.

doi: $10.21037 /$ atm.2020.03.210

View this article at: http://dx.doi.org/10.21037/atm.2020.03.210

Many women with early-stage breast cancers experience bone loss, and some are at increased risk of osteoporosis. The mechanisms are age-related bone loss and estrogen deprivation either through the development of chemotherapy-induced ovarian failure (CIOF) (1), or receiving aromatase inhibitors (2). The third generation amino-bisphosphonate zoledronic acid (ZA), and the receptor activator of nuclear factor- $\kappa \mathrm{B}$ ligand (RANKL) inhibitor, denosumab, effectively prevent or treat osteoporosis in these settings (3). What is less clear is whether bone-modifying agents (BMAs) have clinically significant anti-cancer effects despite data that provides the underlying rationale for testing it in the clinic.

$\mathrm{ZA}$ and denosumab are used to treat skeletal metastases based on a reduction in skeletal-related events such as spinal cord compression, pathological fracture, and the necessity for radiation or surgery to bone (3). Figure 1 explains how these drugs work. The regulation of bone mass involves two levels: at the "macro" level, the control is through systemic hormones (e.g., androgens, estrogens, calcitonin, and parathyroid hormone), and the mechanical forces imposed by gravity. The "micro" level occurs in the bone-remodeling unit, comprised of two primary cell types. Osteoblasts, derived from mesenchymal precursor cells, are responsible for new bone formation, whereas osteoclasts derived from hematopoietic precursor cells are responsible for bone resorption.

The dynamic balance between osteoblast and osteoclast function regulates new bone formation and resorption (4). The osteoblast is the master regulator of bone-remodeling unit secreting both osteoprotegerin (OPG, also called osteoclastogenesis inhibitory factor, a member of the tumor necrosis factor receptor superfamily), and RANKL. When RANKL binds to RANKL receptor located on osteoclast precursor cells, it causes differentiation into mature osteoclasts and stimulates the multiple mechanisms by which bone resorption occurs. The osteoblast also secretes OPG that acts as a decoy receptor for RANKL, thus putting the brakes on osteoclasts. Also, T-cells play critical roles in maintaining bone mass. Estrogen deficiency of normal menopause causes $\mathrm{T}$ cells to secrete tumor necrosis factoralpha and RANKL that activate osteoclasts, causing bone resorption $(5,6)$.

In bone loss and osteoporosis, as well as tumors metastasizing to the bone, osteoclasts are activated, and bone resorption occurs (Figure 1). ZA inhibits osteoclast differentiation, inhibits the binding of the activated osteoclast to the bone surface, and inhibits the lysosomal secretion of hydrochloric acid, one of the major mechanisms of bone resorption. Denosumab is a humanized monoclonal antibody to RANKL. By binding to RANKL, it inhibits osteoclasts.

Experimentally ZA and RANKL inhibition reduce the induce of skeletal metastases in animal models $(7,8)$. Disseminated tumor cells (DTCs) reside in the bone marrow and contribute to other sites of metastases (9). DTCs serve as a prognostic factor in early breast cancer (10), and ZA can reduce DTCs in the human bone marrow $(11,12)$. The preclinical and clinical data support the hypothesis that BMAs have anti-cancer effects, and a series 


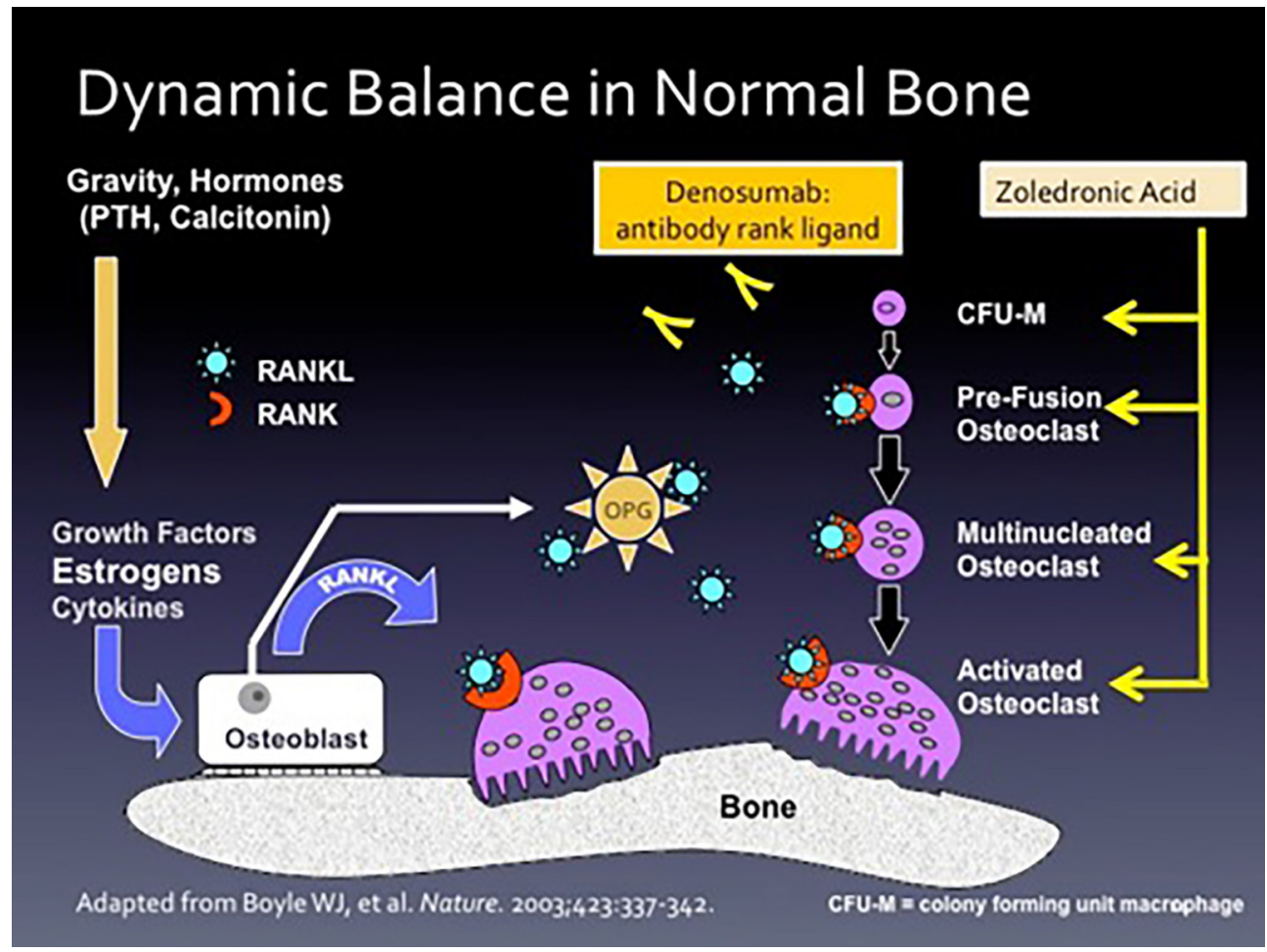

Figure 1 Dynamic balance in normal bone.

of clinical trials tested this hypothesis.

It is instructive to review trials that examined ZA, the Austrian Breast Cancer Study Group (ABCSG) trial 12 (13), AZURE (14), and National Surgical Adjuvant Breast and Bowel (NSAPB) B-34 trials that examined oral clodronate (15). ABCSG trial 12 was the first clinical trial to describe the anti-cancer effects of ZA. Eighteenhundred and three premenopausal women with stages I and II breast cancers received goserelin, a gonadotrophinreleasing hormone $(\mathrm{GnRH})$ antagonist, that rendered them "chemically" postmenopausal. The women were randomized in a $2 \times 2$ factorial design to receive tamoxifen or anastrozole, with or without ZA. The primary endpoint was disease-free survival (DFS). The dose and schedule of ZA was $4 \mathrm{mg}$ IV every six months for three years, or a total of 6 doses. At a median follow-up of nearly eight years, the DFS was $88.4 \%$ and $85.0 \%$, in ZA and control arms, respectively [hazard ratio $(\mathrm{HR})=0.77 ; 95 \%$ CI: 0.60 to $0.99, \mathrm{P}=0.042$ ] There were fewer locoregional $(2.7 \%$ vs. $4.4 \%)$, distant (6.0\% vs. $7.1 \%)$, and bone $(2.7 \%$ vs. $3.5 \%)$ recurrences in the ZA-treated women. The overall survival (OS) was numerically better in ZA treatment (96.7\% vs. 94.5) but not statistically significant with the HR of 0.66 (95\% CI: 0.43 to $1.12, \mathrm{P}=0.06$ ).

The AZURE trial was a phase III open-label of ZA added to standard adjuvant therapy in 3,360 women with stage II and III breast cancer patients. The schedule of ZA was intensive, with 19 doses over five years. Unlike ABCSG trial 12 , there were no differences in DFS and invasive DFS with a median of 63 months follow-up. However, in a preplanned subset analysis, there was a statistically significant interaction with menopausal status. The invasive DFS was $78.2 \%$ vs. $71 \%$ (adjusted HR $=0.75,95 \%$ CI: 0.59 to 0.96 , $\mathrm{P}=0.02$ ), and overall survival was $84.6 \%$ vs. $78.7 \%$ (adjusted $\mathrm{HR}=0.74,95 \%$ CI: 0.55 to $0.98, \mathrm{P}=0.04$ ) in women more than five years postmenopausal. The specific events were less locoregional, second invasive primary breast cancers, and distant recurrences for ZA-treated women. Curiously, distant recurrences in bone did not differ between the postmenopausal treated with ZA and controls.

NSABP B-34 was a phase III randomized, double-blind, placebo-controlled trial using oral clodronate, a less potent first-generation bisphosphonate not available in the US, in 3,324 women ( $65 \%$ over the age of 50 years) receiving adjuvant therapy. The oral clodronate/placebo was $1,600 \mathrm{mg}$ per day for three years. With a median follow-up of 8 years, 
like the AZURE trial, there were no differences in DFS or OS in the entire trial population. However, in women over 50 years old who received clodronate the bone-metastasis free-interval ( $\mathrm{HR}=0.6295 \% \mathrm{CI}$ : 0.40 to 0.95 ), non-bone metastasis-free interval ( $\mathrm{HR}=0.6395 \% \mathrm{CI}: 0.43$ to 0.91 ), and the recurrence-free interval (HR $=0.7595 \%$ CI: 0.57 to $0.99)$ were all statistically significant.

AZURE and NSABP B34 are the largest of the trials of bisphosphonates in the adjuvant setting. Several aspects of these trials raised questions. What about the many premenopausal who went into CIOF? CIOF frequently occurs (40-60\% of women depending on their age when starting chemotherapy) and rapidly (within 6-12 months) in premenopausal women (1). These women would be rendered postmenopausal, yet the anti-cancer effect was not observed in premenopausal women. Considering the underlying mechanism and preclinical data, one would predict that ZA would reduce bone metastases. However, in the AZURE trial using ZA on an intensive schedule akin to the treatment of bone metastases (19 vs. 24 treatments), showed no reduction in bone metastases. Whereas, using less potent oral clodronate in the NASBP B-34 trial, or using ZA on a much less intensive schedule as in the ABCSG trial 12, did show a reduction in bone metastases.

Conflicting results in other trials led the Early Breast Cancer Trialist Collaborative Group (EBCCG) to perform a meta-analysis of seventeen randomized trials of ZA $v s$. control/placebo in over 17,000 women (16). Among the over 6,000 premenopausal women, the effect on disease outcomes was nil. However, among the more 11,000 postmenopausal women, treatment with ZA led to fewer bone recurrences (HR $=0.7295 \%$ CI: 0.60 to $0.86, \mathrm{P}=0.0002$ ), and breast cancer mortality ( $\mathrm{HR}=0.8295 \% \mathrm{CI}: 0.73$ to $0.93, \mathrm{P}=0.002$ ). At tenyears, the absolute reductions in bone recurrences were $2.2 \%$, and in breast cancer mortality were $3.3 \%$.

The National Comprehensive Cancer Center Network (17), American Society of Clinical Oncology (18), European Society of Medical Oncology (19) and St. Gallen's Conference (20) guidelines say "consider" ZA use postmenopausal women, especially in women with a high risk of metastatic disease. Despite these recommendations, only about forty percent of oncologists say they routinely add bisphosphonates to adjuvant therapy solely for the anti-cancer effect, independent of prevention or treatment of osteoporosis (20). Why is this so? The possibilities include it is unclear what is a sufficiently high risk of distant recurrences to justify adding ZA as part of adjuvant therapy. One node-positive, three-nodes positive, or ten- nodes positive? Also, to observe the anti-cancer effects, the number of doses of $\mathrm{ZA}$ is unclear. Six-doses of ZA over three years as in ABCSG trial 12 (13) or nineteen-doses over a five period as in the AZURE trial (14)? Finally, why should the effect only work in a low estrogen environment?

BMA agents have side-effects too, the most serious of which is the dose and duration-dependent osteonecrosis (21). Osteonecrosis is most often observed in the treatment of metastatic bone disease, where the rates for both $\mathrm{ZA}$ and denosumab are in the 1-2\% range. In the AZURE trial using an intensive schedule of $\mathrm{ZA}$, the cumulative percentage of osteonecrosis was $1.1 \%$ (14). Whereas in the ABCSG trial 12 , osteonecrosis was not observed.

Now let's turn to the data supporting denosumab as an anti-cancer drug. First, in the treatment of bone metastases, denosumab was superior to $\mathrm{ZA}$ in time to first and subsequent skeletal-event analysis $(22,23)$. Although, some have questioned the superiority denosumab over $\mathrm{ZA}$ as there was no difference in survival nor some of the individual skeletal-related events (24). Also, denosumab was much less cost-effective than ZA (25). The ease of administration via the subcutaneous route and "superiority" in the treatment of bone metastases may be responsible for denosumab's broad acceptance by the oncologic community.

The Austrian Breast Cancer Study Group (ABCSG) trial 18 is instructive to review (26). ABCSG trial 18 was a double-blind, placebo-controlled trial of denosumab/ placebo in 3,425 postmenopausal women receiving an aromatase inhibitor. The primary endpoint was a reduction in fractures. Exploratory analyses were disease-free and bone metastases-free survival. Notably, the schedule of denosumab was every 6 months for five years or a total of 10 treatments. The primary endpoint was met, with a median of 73 months, the secondary endpoint of DFS was better on denosumab arm with HR of 0.82 (95\% CI: 0.69 to 0.98$)$. To make the hazard rate more clinically relevant, at eight years, the improvement in DFS was an absolute 3\% difference (80.6\% versus $77.5 \%)$.

Looking at the specific events in trial ABCSG trial 18, there were no differences in histologically-verified invasive local-regional recurrences, distant recurrences, contralateral new primary cancers, or ductal carcinoma in situ. The superior DFS in the denosumab-treated women was the non-histologically verified distant metastases or new primary breast cancers. One could argue that the doubleblind, placebo-controlled design, and the intention-totreat analysis, would minimize bias. Still, possibly chance or unknown imbalances existed to make the numbers of 
histologically unverified new primary breast cancers (100 $v s .80$ ) and distant recurrences (68 vs. 56) higher in placebotreated patients.

With the above as background, the recently published Amgen-sponsored, double-blind, placebo-controlled adjuvant denosumab in early breast cancer or D-CARE trial comes into view (27). The primary hypothesis was denosumab added to standard of care adjuvant, or neoadjuvant therapy would improve bone metastasis-free survival over that of standard care therapy plus placebo. The event-rate, as in all phase III clinical breast cancer trials, was lower than predicted. Rather than increase the sample size, the sponsors decided to change the trial analysis from an event-driven analysis (where the events were bone metastasis) to a time-driven analysis when all the trial participants completed a minimum of 5 years of follow-up.

From 2010 to 2012, 4,509 women were randomized. The median age was 50 (range, 44-59), 54\% were postmenopausal, $93 \%$ were $\mathrm{N} 1-\mathrm{N} 3$, and the trial included all breast cancer subtypes in the expected frequencies. The schedule of denosumab was intensive, initially monthly for six months, then every three months for 4.5 years, and the median number of doses received was 25 (range, 1-26) out of a planned 26 treatments. The primary endpoint, bone recurrences was not different for denosumab and placebo groups with the hazard ratio (HR) 0.97 (95\% CI: 0.82 to 1.14), $\mathrm{P}=0.70$, and the secondary endpoint, disease-free survival was not different (HR 1.04; 95\% CI: 0.91 to 1.19 , $\mathrm{P}=0.57$ ). They presented the results of a pre-planned subset analysis, and there were no differences by menopausal status.

With the uncertainties of ABCSG trial and wholly negative D-CARE trial, denosumab should not be used as an anti-cancer drug. There are several ongoing trials of denosumab as an anti-cancer drug in the neoadjuvant setting, in aromatase inhibitor-induced bone loss, or the schedule of administration that are either fully enrolled but not reported as yet or actively recruiting (http:// ClinicalTrials.gov). Hopefully, more information may be forthcoming. In terms of ZA, data are supporting its' use as an anti-cancer drug. However, several outstanding questions remain, including which specific population of high-risk postmenopausal women and the schedule of administration. However, it is unlikely that future trials will be dedicated to answering these questions.

Different oncologists will interpret the data described above in different ways. In the low risk, estrogen receptorpositive, node-negative postmenopausal women, or women rendered postmenopausal by treatment with a $\mathrm{GnRH}$ agonist, the benefits of ZA maybe not justified because of a favorable prognosis. In contrast, in high-risk, multiply node-positive, higher-stage women, the data on ZA may be compelling. For women with breast cancer who at risk of developing osteoporosis or have established osteoporosis, using ZA every 6 months will build bone and improve the T-scores and may help reduce recurrences in bone and improve mortality.

\section{Acknowledgments}

Funding: None.

\section{Footnote}

Conflicts of Interest: The author has completed the ICMJE uniform disclosure form (available at http://dx.doi. org/10.21037/atm.2020.03.210). The author has no conflicts of interest to declare.

Ethical Statement: The author is accountable for all aspects of the work in ensuring that questions related to the accuracy or integrity of any part of the work are appropriately investigated and resolved.

Open Access Statement: This is an Open Access article distributed in accordance with the Creative Commons Attribution-NonCommercial-NoDerivs 4.0 International License (CC BY-NC-ND 4.0), which permits the noncommercial replication and distribution of the article with the strict proviso that no changes or edits are made and the original work is properly cited (including links to both the formal publication through the relevant DOI and the license). See: https://creativecommons.org/licenses/by-nc-nd/4.0/.

\section{References}

1. Shapiro CL, Manola J, Leboff M. Ovarian failure after adjuvant chemotherapy is associated with rapid bone loss in women with early-stage breast cancer. J Clin Oncol 2001;19:3306-11.

2. Lustberg MB, Reinbolt RE, Shapiro CL. Bone health in adult cancer survivorship. J Clin Oncol 2012;30:3665-74.

3. Shapiro CL, Van Poznak C, Lacchetti C, et al. Management of Osteoporosis in Survivors of Adult Cancers With Nonmetastatic Disease: ASCO Clinical Practice Guideline. J Clin Oncol 2019;37:2916-46.

4. Boyle WJ, Simonet WS, Lacey DL. Osteoclast 
differentiation and activation. Nature 2003;423:337-42.

5. Weitzmann MN, Pacifici R. T cells: unexpected players in the bone loss induced by estrogen deficiency and in basal bone homeostasis. Ann N Y Acad Sci 2007;1116:360-75.

6. D'Amelio P, Grimaldi A, Di Bella S, et al. Estrogen deficiency increases osteoclastogenesis up-regulating $T$ cells activity: a key mechanism in osteoporosis. Bone 2008;43:92-100.

7. Beuzeboc P, Scholl S. Prevention of Bone Metastases in Breast Cancer Patients. Therapeutic Perspectives. J Clin Med 2014;3:521-36.

8. Canon JR, Roudier M, Bryant R, et al. Inhibition of RANKL blocks skeletal tumor progression and improves survival in a mouse model of breast cancer bone metastasis. Clin Exp Metastasis 2008;25:119-29.

9. Shiozawa Y, Eber MR, Berry JE, et al. Bone marrow as a metastatic niche for disseminated tumor cells from solid tumors. Bonekey Rep 2015;4:689.

10. Domschke C, Diel IJ, Englert S, et al. Prognostic value of disseminated tumor cells in the bone marrow of patients with operable primary breast cancer: a long-term followup study. Ann Surg Oncol 2013;20:1865-71.

11. Aft R, Naughton $M$, Trinkaus K, et al. Effect of zoledronic acid on disseminated tumour cells in women with locally advanced breast cancer: an open label, randomised, phase 2 trial. Lancet Oncol 2010;11:421-8.

12. Banys M, Solomayer EF, Gebauer G, et al. Influence of zoledronic acid on disseminated tumor cells in bone marrow and survival: results of a prospective clinical trial. BMC Cancer 2013;13:480.

13. Gnant M, Mlineritsch B, Stoeger H, et al. Zoledronic acid combined with adjuvant endocrine therapy of tamoxifen versus anastrozol plus ovarian function suppression in premenopausal early breast cancer: final analysis of the Austrian Breast and Colorectal Cancer Study Group Trial 12. Ann Oncol 2015;26:313-20.

14. Coleman RE, Marshall H, Cameron D, et al. Breastcancer adjuvant therapy with zoledronic acid. N Engl J Med 2011;365:1396-405.

15. Paterson AH, Anderson SJ, Lembersky BC, et al. Oral clodronate for adjuvant treatment of operable breast cancer (National Surgical Adjuvant Breast and Bowel Project protocol B-34): a multicentre, placebo-controlled, randomised trial. Lancet Oncol 2012;13:734-42.

16. Early Breast Cancer Trialists' Collaborative G, Coleman R, Powles T, et al. Adjuvant bisphosphonate treatment in early breast cancer: meta-analyses of individual patient data from randomised trials. Lancet 2015;386:1353-61.

17. Gradishar WJ, Anderson BO, Balassanian R, et al. NCCN
Guidelines Insights: Breast Cancer, Version 1.2017. J Natl Compr Canc Netw 2017;15:433-51.

18. Dhesy-Thind S, Fletcher GG, Blanchette PS, et al. Use of Adjuvant Bisphosphonates and Other Bone-Modifying Agents in Breast Cancer: A Cancer Care Ontario and American Society of Clinical Oncology Clinical Practice Guideline. J Clin Oncol 2017;35:2062-81.

19. Cardoso F, Kyriakides S, Ohno S, et al. Early breast cancer: ESMO Clinical Practice Guidelines for diagnosis, treatment and follow-updagger. Ann Oncol 2019;30:1194-220.

20. Balic M, Thomssen C, Wurstlein R, et al. St. Gallen/ Vienna 2019: A Brief Summary of the Consensus Discussion on the Optimal Primary Breast Cancer Treatment. Breast Care (Basel) 2019;14:103-10.

21. Yarom N, Shapiro CL, Peterson DE, et al. MedicationRelated Osteonecrosis of the Jaw: MASCC/ISOO/ ASCO Clinical Practice Guideline. J Clin Oncol 2019;37:2270-90.

22. Stopeck AT, Lipton A, Body JJ, et al. Denosumab compared with zoledronic acid for the treatment of bone metastases in patients with advanced breast cancer: a randomized, doubleblind study. J Clin Oncol 2010;28:5132-9.

23. Lipton A, Fizazi K, Stopeck AT, et al. Superiority of denosumab to zoledronic acid for prevention of skeletal-related events: a combined analysis of 3 pivotal, randomised, phase 3 trials. Eur J Cancer 2012;48:3082-92.

24. Shapiro CL, Moriarty JP, Borah B. Reply to L. Kennedy et al. J Clin Oncol 2018;36:1052-3.

25. Shapiro CL, Moriarty JP, Dusetzina S, et al. CostEffectiveness Analysis of Monthly Zoledronic Acid, Zoledronic Acid Every 3 Months, and Monthly Denosumab in Women With Breast Cancer and Skeletal Metastases: CALGB 70604 (Alliance). J Clin Oncol 2017;35:3949-55.

26. Gnant M, Pfeiler G, Steger GG, et al. Adjuvant denosumab in postmenopausal patients with hormone receptor-positive breast cancer (ABCSG-18): disease-free survival results from a randomised, double-blind, placebocontrolled, phase 3 trial. Lancet Oncol 2019;20:339-51.

27. Coleman R, Finkelstein DM, Barrios C, et al. Adjuvant denosumab in early breast cancer (D-CARE): an international, multicentre, randomised, controlled, phase 3 trial. Lancet Oncol 2020;21:60-72.

Cite this article as: Shapiro CL. Caring about bone-modifying agents (BMAs) in women with early-stage breast cancer. Ann Transl Med 2020;8(16):1038. doi: 10.21037/atm.2020.03.210 